\title{
A Novel Low Temperature Curable Photosensitive Polybenzoxazole
}

\author{
Fumihiro TOYOKAwA, Yuji SHIBASAKI, and Mitsuru UEDA ${ }^{\dagger}$
}

Department of Organic and Polymeric Materials, Graduate School of Science and Engineering,
Tokyo Institute of Technology, 2-12-1-H120 O-okayama, Meguro-ku, Tokyo 152-8552, Japan

(Received January 24, 2005; Accepted April 19, 2005; Published July 15, 2005)

\begin{abstract}
A novel positive-tone photosensitive polybenzoxazole for low temperature cyclization has been developed. The matrix polymer (PHA-6F) was prepared from 4,4'-(hexafluoroisopropylidene)bis $(o$-aminophenol) (6FAP) and 4,4'-oxybis(benzoyl chloride) (OBBC) by low temperature solution polycondensation, and the photosensitive polymer was formulated with PHA-6F, a dissolution inhibitor 1-\{1,1-bis[4-(2-diazo-1-(2H)naphthalenone-4-sulfonyloxy)phenyl]ethyl\}-4-\{1-[4-(2-diazo-1(2H)naphthalenone-4-sulfonyloxy)phenyl]methylethyl $\}$ benzene (S-DNQ), and a photo- and thermoacid generator (5-propylsulfonyloxyimino-5H-thiophen-2-ylidene)-2-(methylphenyl)acetonitrile (PTMA). The photosensitivity and contrast of the $2.4 \mu \mathrm{m}$ thick film were $15 \mathrm{~mJ} / \mathrm{cm}^{2}$ and 2.5 , respectively. A clear positive polybenzoxazole image featuring $8 \mu \mathrm{m}$ line-and-space pattern was obtained, when a $2.1 \mu \mathrm{m}$-photosensitive film containing PHA-6F, S-DNQ, and PTMA (15:3:2 in weight ratio) was prebaked at $120^{\circ} \mathrm{C}$ for 5 min, irradiated with $365 \mathrm{~nm}$ light $\left(60 \mathrm{~mJ} / \mathrm{cm}^{2}\right)$, developed with an $2.38 \mathrm{wt} \%$ tetramethylammonium hydroxide (TMAH) solution for $90 \mathrm{~s}$, washed with acetic acid, and cured at $250{ }^{\circ} \mathrm{C}$ for $10 \mathrm{~min}$. [DOI $10.1295 /$ polymj.37.517]

KEY WORDS Photosensitive Polybenzoxazole / Low Temperature Solid-phase Cyclization / $i$-Line Lithography / Poly (o-hydroxyamine) / Alkaline Developer / Thermoacid Generator /
\end{abstract}

Polybenzoxazoles (PBOs) have been used as protection and insulation layers for very large scale integration circuits (VLSI) and multi-chip modules for computers owing to their high thermal stability and excellent mechanical property. ${ }^{1-4}$ Photosensitive PBOs (PSPBOs) ${ }^{5-21}$ have been developed to simplify the processing steps because phenolic hydroxyl groups in precursors of PBOs provide adequate solubility toward an aqueous alkaline developer. Up to now PSPBO based on the poly $(o$-hydroxyamide) (PHA6F) derived from 6FAP and 4,4'-oxybis(benzoic acid) derivatives ${ }^{22,23}$ with a photosensitive compound has been widely used, in which the image of PHA-6F is finally converted into that of $\mathrm{PBO}$ by thermal treatment at $350{ }^{\circ} \mathrm{C}$. This high-temperature process is hardly applicable to the conventional electronic applications containing at least one or more thermally unstable organic components. Therefore, a novel method for the formation of PBO patterning at low temperature curing is desired.

Recently sulfonic acids were found to work as an effective catalyst for low temperature cyclization of PHA. ${ }^{24}$ Especially, PTMA, which is known as an excellent photoacid generator with high quantum yield at $436 \mathrm{~nm}$ wavelength, ${ }^{25}$ lowered the cyclization temperature at $250{ }^{\circ} \mathrm{C},{ }^{26}$ significantly lower than noncatalytic cyclization at $350^{\circ} \mathrm{C}$. Thus, the development of low temperature cyclization system in a photosensitive PBO would be useful for expanding its applica-
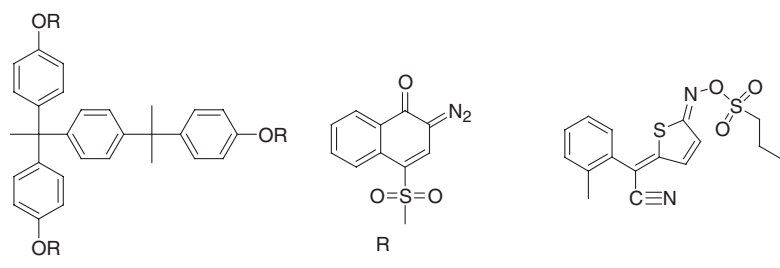

S-DNQ

PTMA

Scheme 1 .

tion to various fields. PTMA can also be used as a thermoacid generator, ${ }^{26}$ and it will functions as an acid catalyst for the cyclization by heating after development.

We describe here a novel photosensitive $\mathrm{PBO}$ consisting of PHA-6F, a dissolution inhibitor S-DNQ, and a photo- and thermoacid generator PTMA (Scheme 1), enabling low temperature solid-phase cyclization of PHA-6F after the patterning.

\section{EXPERIMENTAL}
Materials
6FAP was recrystallized from tetrahydrofuran (THF)/hexane. 4,4'-Oxybis(benzoyl chloride) (OBBC) was prepared from the corresponding acid with thi- onyl chloride. 1-Methyl-2-pyrrolidinone (NMP) was purified by vacuum distillation from calcium hydride and stored over $4 \AA$ molecular sieves. PTMA and 
S-DNQ were kindly supplied from Ciba Specialty Chemicals and Sumitomo Bakelite Co. Ltd., respectively, and used as received. PHA-6F was prepared from 6FAP and OBBC in NMP by low temperature solution polycondensation in $93 \%$ yield $\left(M_{\mathrm{n}}=\right.$ $25000, M_{\mathrm{w}} / M_{\mathrm{n}}=1.6$ determined by GPC (DMF, PSt standards)). Other reagents and solvents were used as received.

\section{Dissolution Rate}

All baking processes were performed on a hot plate with a digital thermo-controller. PTMA (10-30 wt \% to PHA-6F) and S-DNQ (5-20 wt \% to PHA-6F) were dissolved in PHA-6F solution in $N, N$-dimethylacetamide (DMAc) (20 wt \% of PHA-6F) to construct a photosensitive polymer. The polymer film spin-cast on a silicon wafer was prebaked at $80-120^{\circ} \mathrm{C}$ for $5 \mathrm{~min}$, exposed to a filtered super-high-pressure mercury lamp at $365 \mathrm{~nm}$ wavelength, and developed with a $2.38 \mathrm{wt} \% \mathrm{TMAH}$ solution at $25^{\circ} \mathrm{C}$.

\section{Photosensitivity}

A $2.4 \mu \mathrm{m}$-thick polymer film containing PHA-6F, S-DNQ, and PTMA (15:3:2 in weight ratio) on a silicon wafer was prebaked at $120^{\circ} \mathrm{C}$ for $5 \mathrm{~min}$, exposed to $365 \mathrm{~nm}$ light, and developed with a $2.38 \mathrm{wt} \%$ TMAH solution at $25^{\circ} \mathrm{C}$ for $300 \mathrm{~s}$. A characteristic curve was obtained by plotting a normalized film thickness against exposure energy.

\section{Evaluation of Cyclization}

The degree of cyclization was determined by Infrared (IR) spectroscopy, in which the absorption change at 1051 (C-O stretch of benzoxazole) and $1250 \mathrm{~cm}^{-1}$ (C-O stretch of phenyl ether) was monitored. No influence on these two absorptions by the catalyst and solvent was assumed.

\section{Measurements}

IR spectra were recorded on a Horiba FT-210 spectrophotometer. Thermal analyses were performed on a Seiko EXSTAR 6000 TG/DTA 6300 thermal analyzer at heating rate of $10{ }^{\circ} \mathrm{C} / \mathrm{min}$ for thermogravimetry (TG). Number- and weight-average molecular weights $\left(M_{\mathrm{n}}\right.$ and $\left.M_{\mathrm{w}}\right)$ were determined by a gel permeation chromatography (GPC) with polystyrene calibration using Tosoh HLC-8120 system equipped with a consecutive polystyrene gel column (TSK GELs, $\mathrm{GMH}_{\mathrm{HR}}-\mathrm{M}$ and $\mathrm{GMH}_{\mathrm{HR}}-\mathrm{L}$ ) at $40^{\circ} \mathrm{C}$ in $\mathrm{DMF}$ containing $0.01 \mathrm{M}$ of lithium bromide at a flow rate of $1.0 \mathrm{~mL} / \mathrm{min}$. Film thickness was measured by a Dektak $^{3}$ surface profiler (Veeco Instrument Inc). Scanning electron micrograph (SEM) was taken by Technex Lab Co., Ltd. Tiny SEM 1540.

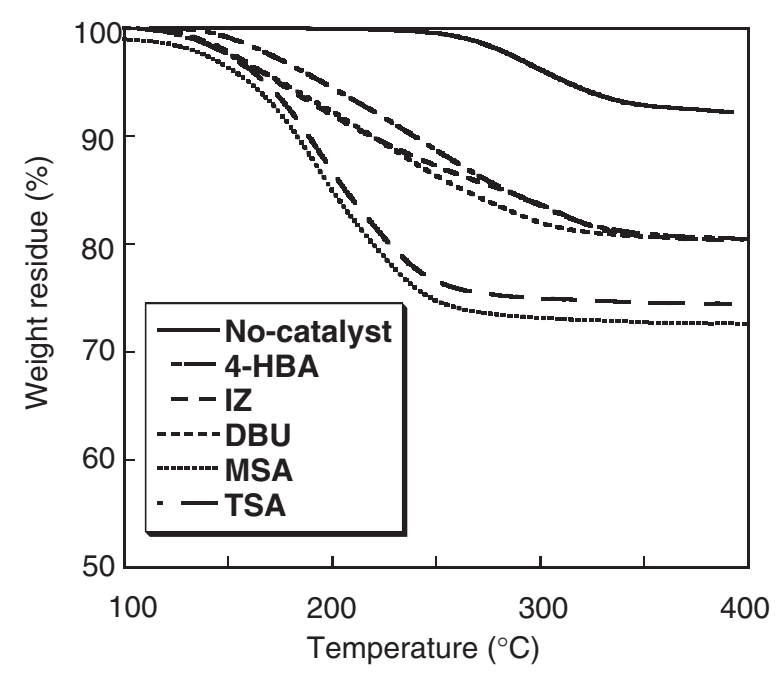

Figure 1. TG profiles of PHA-6F films without (solid line) and with $5 \mathrm{wt} \%$ additives of 4-hydroxybenzoic acid (4-HBA) (dashed-dotted line), imidazole (IZ) (short-dashed line), diazabicyclo[5.4.0] undec-7-ene (DBU) (broken line), $p$-toluenesulufonic acid (TSA) (long-dashed line), and methanesulfonic acid (MSA) (dotted line).

\section{RESULTS AND DISCUSSION}

\section{Cyclization Catalyst}

Several acid and base catalysts such as 4-hydroxybenzoic acid (4-HBA), imidazole (IZ), diazabicyclo[5.4.0] undec-7-ene (DBU), p-toluenesulfonic acid (TSA), and methanesulfonic acid (MSA) were employed to investigate the effect for cyclization of PHA-6F to PBO. Figure 1 illustrates the TG $\left(10^{\circ} \mathrm{C} /\right.$ min under nitrogen) profiles in each sample. Without any additives, PHA-6F starts to decrease its weight from $250^{\circ} \mathrm{C}$, and the cyclization finishes at $350^{\circ} \mathrm{C}$. This weight loss of $6 \%$ agreed well with the calculated value of $5.8 \%$ if the water was completely removed during the cyclization. Sulfonic acids are more effective than the other catalysts such as benzoic acid and base catalysts, and the cyclization temperature can be lowered to $250^{\circ} \mathrm{C}$. Thus, as a thermoacid generator for the present photosensitive polymer system, sulfonic acid derivatives were chosen. PTMA was effective to lower the transformation temperature from PHA into $\mathrm{PBO}$ by protonation of carbonyl oxygen resulting in easy nucleophilic attack of phenol oxygen on amido carbonyl, ${ }^{26}$ thus from the results mentioned above sulfonic acid generated from PTMA is a promoter, and we expected PTMA could play as a photoacid generator and promoter for fabrication of PBO image.

\section{Lithographic Evaluation}

PTMA is known as a dissolution inhibitor as well as a photoacid generator, thus a photosensitive polymer was constructed with PHA-6F and PTMA. The effect 


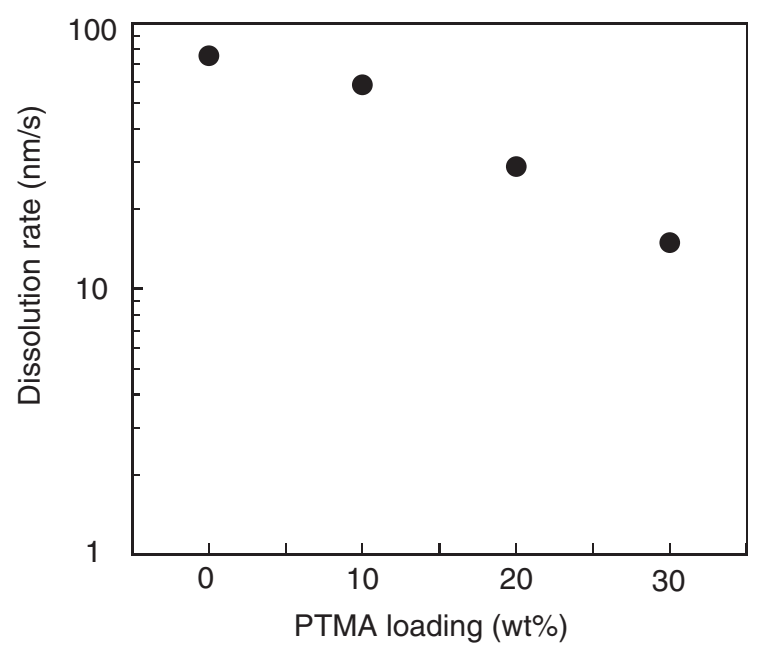

Figure 2. Effect of PTMA loading on dissolution rate $(\mathrm{nm} / \mathrm{s})$. Conditions; PTMA (0-30wt \% to PHA-6F), DMAc solution (20 wt $\%$ of PHA-6F), prebaking at $120^{\circ} \mathrm{C}$ for $5 \mathrm{~min}$, development $2.38 \mathrm{wt} \%$ TMAH solution at $25^{\circ} \mathrm{C}$.

of PTMA loading on the dissolution rate of the film was investigated as shown in Figure 2. The dissolution rate was estimated by the measurement of the film thickness after development. Although increasing the amounts of PTMA up to $30 \mathrm{wt} \%$ to PHA-6F, the dissolution contrast between the PTMA un-loaded and loaded films is small probably because of its small size. Thus, the conventional dissolution inhibitor S-DNQ was decided to add in this system. The effect of S-DNQ loading on the dissolution rate of the polymer film was studied in similar conditions described in Figure 2. The PHA-6F film (containing $10 \mathrm{wt} \%$ PTMA to PHA-6F) spin-cast on a silicon wafer was irradiated with $365 \mathrm{~nm}$ light at $100 \mathrm{~mJ} / \mathrm{cm}^{2}$, and developed with a $2.38 \mathrm{wt} \% \mathrm{TMAH}$ solution at $25^{\circ} \mathrm{C}$. The dissolution rate decreases with increasing the amounts of S-DNQ, and becomes zero with $15 \mathrm{wt} \%$ loading, where a large dissolution contrast between the exposed and unexposed areas is achieved as shown in Figure 3. This effective dissolution inhibition of S-DNQ attributes its bulky structure compared with PTMA. S-DNQ absorbs light, resulting in its transformation into indencarboxilic acid which accelerates solubility in alkaline developer. Prebaking conditions are important because they generally affect the dissolution rate of the film due to the residual solvent. Thus, the effect of prebaking temperature from 80$120^{\circ} \mathrm{C}$ for $5 \mathrm{~min}$ was studied (Figure 4 ). Baking at $120^{\circ} \mathrm{C}$ for $5 \mathrm{~min}$ effectively reduces the dissolution rate. Thus, the suitable prebaking temperature was determined to be $120^{\circ} \mathrm{C}$.

After these preliminary optimization studies involving S-DNQ loading and prebaking temperature, the photosensitive polymer was formulated using PHA6F (20 wt \% in DMAc), PTMA (10 wt \% to PHA-6F),

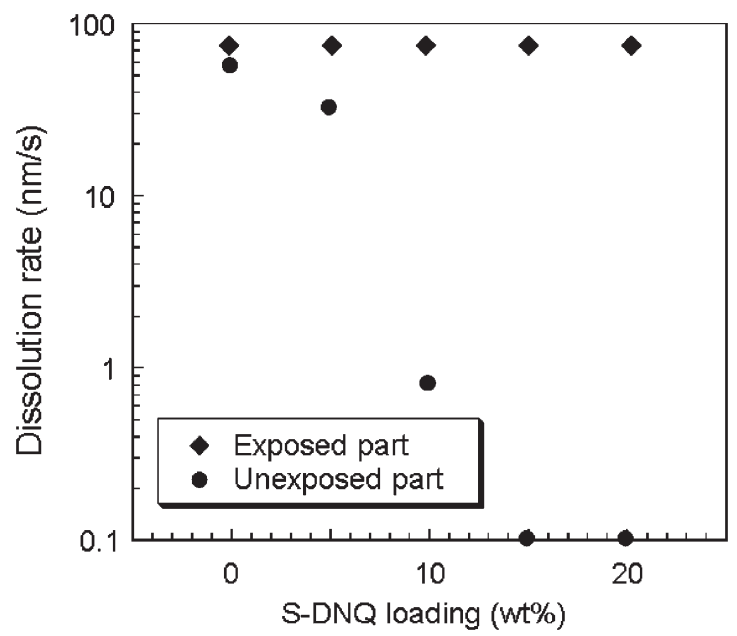

Figure 3. Effect of S-DNQ loading on dissolution rate $(\mathrm{nm} / \mathrm{s})$. Conditions; PTMA (10 wt \% to PHA-6F), S-DNQ (0-20 wt \% to PHA-6F), DMAc solution (20 wt $\%$ of PHA-6F), prebaking at $120^{\circ} \mathrm{C}$ for $5 \mathrm{~min}$, development $2.38 \mathrm{wt} \% \mathrm{TMAH}$ solution at $25^{\circ} \mathrm{C}$.

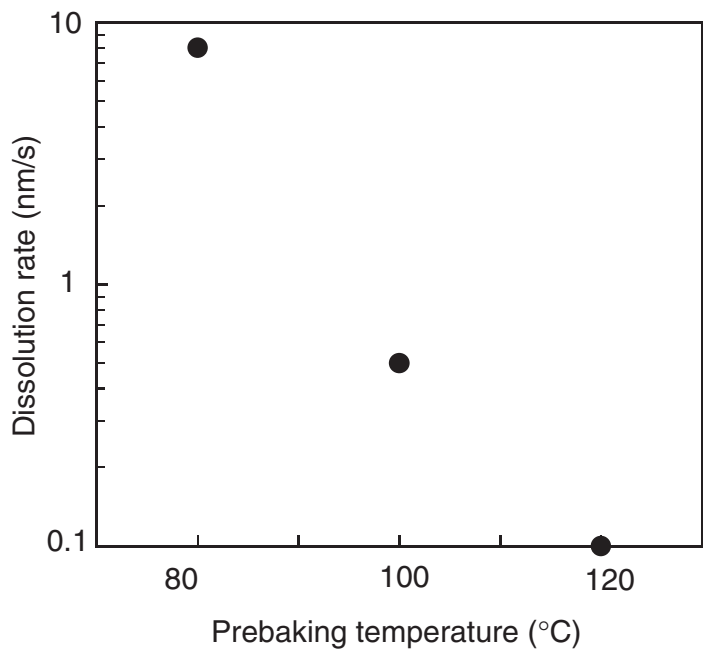

Figure 4. Effect of baking temperature on dissolution rate $(\mathrm{nm} / \mathrm{s})$. Conditions; PTMA (10 wt $\%$ to PHA-6F), S-DNQ (15 wt $\%$ to PHA-6F) DMAc solution (15 wt \% of PHA-6F), prebaking at $80-120^{\circ} \mathrm{C}$ for $5 \mathrm{~min}$, development $2.38 \mathrm{wt} \%$ TMAH solution at $25^{\circ} \mathrm{C}$.

S-DNQ (15 wt \% to PHA-6F). To evaluate the sensitivity and contrast of the photosensitive film, the film thickness after development is plotted as a function of irradiation dose as shown in Figure 5. The sensitivity and contrast was determined to be $15 \mathrm{~mJ} / \mathrm{cm}^{2}$ and 2.5 , respectively, when $2.4 \mu \mathrm{m}$ thick film containing PHA6F, S-DNQ, and PTMA (15:3:2 in weight ratio) was irradiated with $365 \mathrm{~nm}$ light, and developed with a 2.38 wt \% TMAH solution for $300 \mathrm{~s}$.

To realize complete transformation of PHA-6F image into $\mathrm{PBO}$ at low curing temperature (Scheme 2), where the acid catalyst should work well, the $2.1 \mu \mathrm{m}$ photosensitive film containing PHA-6F, S-DNQ, and 


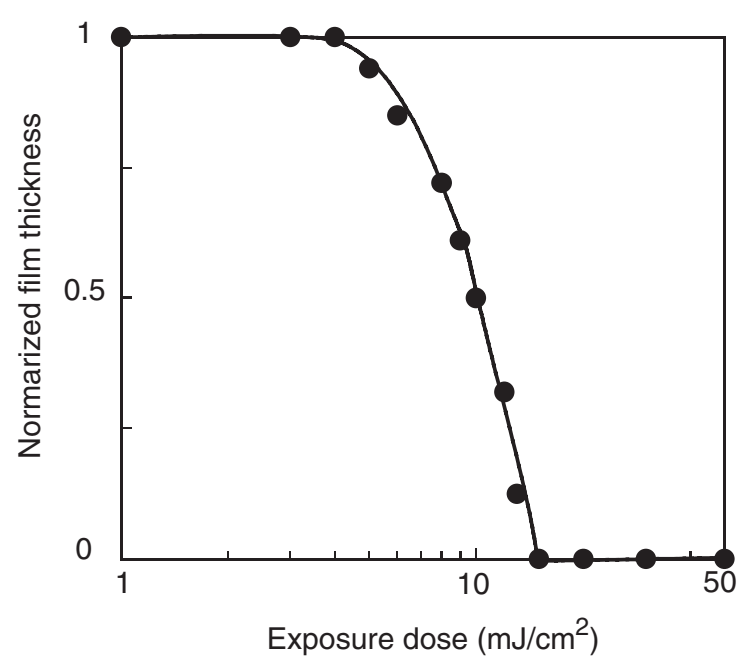

Figure 5. A photosensitivity curve of PHA-6F based photosensitive polymer.

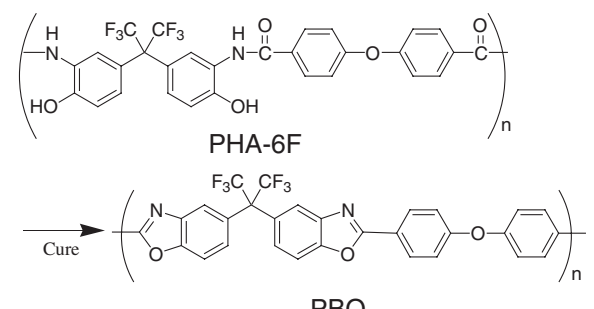

Scheme 2.

PTMA (15:3:2 in weight ratio) was prebaked at $120^{\circ} \mathrm{C}$ for $5 \mathrm{~min}$, irradiated with $365 \mathrm{~nm}$ light $(60$ $\mathrm{mJ} / \mathrm{cm}^{2}$ ), developed with a $2.38 \mathrm{wt} \%$ TMAH solution for $90 \mathrm{~s}$. The remaining alkaline developer was first removed by rinsing the PHA-6F image with an acetic acid aqueous solution for $90 \mathrm{~s}$, and the image was postbaked. Figure 6 shows IR spectra of PSPBO films (A) after prebaking (B) after postbaking at $250{ }^{\circ} \mathrm{C}$ for 10 min (rinsing with acetic acid) and (C) of corresponding PBO film. The characteristic absorption at $1650 \mathrm{~cm}^{-1}$ corresponding to amide stretching was disappeared and a new absorption at 1051 (oxazole $\mathrm{C}-\mathrm{O}$ stretching) was observed when the film was postbaked at $250^{\circ} \mathrm{C}$ for $10 \mathrm{~min}$. Figure 7 depicts a scanning electron micrograph of a contact printed image of the film cured at $250^{\circ} \mathrm{C}$ for $10 \mathrm{~min}$ after development. Although the image after $250^{\circ} \mathrm{C}$ curing shows rounded edges compared with PHA-6F image, a clear PBO image featuring $8 \mu \mathrm{m}$ line-and-space was observed without serious deformation.

\section{CONCLUSIONS}

A novel low curable PSPBO was formulated using PHA-6F (75 wt \%), S-DNQ (15 wt \%), and PTMA $(10 \mathrm{wt} \%)$. The positive-tone image featuring $8 \mu \mathrm{m}$

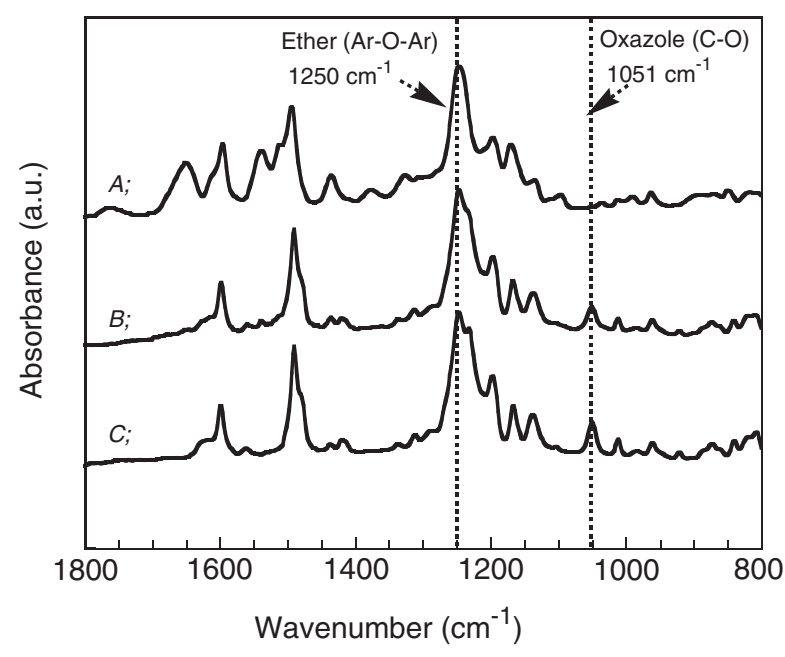

Figure 6. IR spectra of PSPBO films (A) after prebaking (B) after postbaking at $250^{\circ} \mathrm{C}$ for $10 \mathrm{~min}$ (rinsing with acetic acid) and (C) of corresponding PBO film.

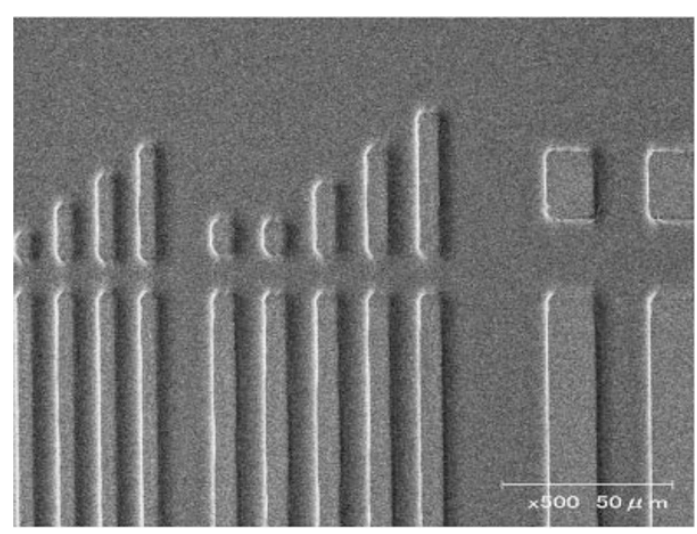

Figure 7. SEM image of PSPBO.

line-and-space was obtained by $365 \mathrm{~nm}$ exposures followed by development with a $2.38 \mathrm{wt} \% \mathrm{TMAH}$ solution. The PHA-6F image was successfully converted into $\mathrm{PBO}$ image at as low as $250^{\circ} \mathrm{C}$ for 10 min. This photo-patterning system with low temperature curing for the formation of PBO image is a promising method and will be applied for practical use.

\section{REFERENCES}

1. T. Kubota and R. Nakanishi, J. Polym. Sci. Part B: Polym. Lett., 2, 655 (1964).

2. Y. Imai, I. Taoka, K. Uno, and Y. Iwakura, Macromol. Chem., 83, 167 (1965).

3. C. Arnold, Jr., J. Polym. Sci., Macromol. Rev., 14, 265 (1979).

4. G. Maiyer, Prog. Polym. Sci., 26, 3 (2001).

5. R. Rubner, A. Hammerschmidt, R. Leuschner, and H. Ahne, in "Polym. Microelectron, Proc. Int. Symp," Y. Tabata, Ed., Kodansha, Tokyo, 1990, p 789.

6. H. Ahne, A. Hammerschmidt, and E. Schmidt, Proceedings of Polym. Mater. Sci. Eng. Am. Chem. Soc., 60, 629 (1989). 
7. H. Ahne, R. Rubner, and R. Sezi, Appl. Surf. Sci., 106, 311 (1996).

8. D. F. Huang, J. Y. Chiou, C. C. Hung, and B. R. Young, Proc. SPIE, 4277, 375 (2001).

9. R. Sezi, A. Maltenberger, W. Radlik, G. Schmid, A. Weber, and K. Buschick, Proc. SPIE, 3582, 236 (1998).

10. H. Makabe, T. Banba, and T. Hirano, J. Photopolym. Sci. Technol., 10, 307 (1997).

11. T. Yamaoka, N. Nakajima, K. Koseki, and Y. Maruyama, J. Polym. Sci., Part A: Polym. Chem., 28, 2517 (1990).

12. D. N. Khanna, Polym. Prepr., Am. Chem. Soc., Div. Polym. Chem., 1, 348 (1990).

13. D. N. Khanna and W. H. Mueller, Polym. Eng. Sci., 29, 954 (1989).

14. R. Rubner, Adv. Mater., 2, 452 (1990).

15. K. Ebara, Y. Shibasaki, and M. Ueda, J. Polym. Sci., Part A: Polym. Chem., 40, 3399 (2002).

16. K. Ebara, Y. Shibasaki, and M. Ueda, Polymer, 44, 333 (2003).

17. K. Ebara, Y. Shibasaki, and M. Ueda, J. Photopolym. Sci.
Tecnol., 16, 287 (2003).

18. C. S. Hong, M. Jikei, and M. Kakimoto, Polym. J., 35, 586 (2003).

19. K. Fukukawa, Y. Shibasaki, and M. Ueda, Polym. J., 36, 489 (2004).

20. K. Fukukawa, Y. Shibasaki, and M. Ueda, Macromolecules, 37, 8256 (2004).

21. K. Fukukawa, Y. Shibasaki, and M. Ueda, Polym. J., 37, 74 (2005).

22. M. D. Houtz, J. M. Lavoie, D. L. Pedrick, E. G. Jones, M. R. Unroe, and L. S. Tan, Polym. Prepr., 35, 437 (1994).

23. W. D. Joseph, J. C. Abed, T. H. Yoon, and J. E. McGrath, Polym. Prepr., Am. Chem. Soc., Div. Polym. Chem., 35, 551 (1994).

24. T.-K. Kim, K.-Y. Choi, K.-S. Lee, D.-W. Park, and M.-Y. Jin, Polym. Bull., 44, 55 (2000).

25. T. Asakura, H. Yamato, and M. Ohwa, J. Photopolym. Sci. Technol., 13, 223 (2000).

26. F. Toyokawa, Y. Shibasaki, and M. Ueda, Chem. Lett., 1342 (2004). 